Objectives: To explore the impact of diacerein and moderate mechanical stimulation on the anabolic metabolism of OA and non-OA chondrocytes, as well as on the integrin-FAK-MAPKs signal transduction cascade in these cells.

Methods: Mechanical stimulation was applied in terms of three different intensities by the Flexcell tension system. Influence on catabolic parameters such as MMPs, ADAMTS, and IL- 6 were assessed by qPCR. Changes in phosphorylation of FAK, STAT3 as well as MAP kinases were verified by western blot analysis. Intracellular calcium was measured fluorimetrically using fura-2.

Results: Mechanical stimulation at moderate intensity (SM/SA) proved to be most efficient in terms of reducing production of matrix degrading enzyme and IL6 expression. Treatment with diacerein by itself and diacerein in combination with SM/SA reduced phosphorylation of FAK and STAT3, which is more pronounced in OA cells. Pretreatment with diacerein for 7 days resulted in an increase in the sensitivity to Yoda1, the agonist for the mechanically activated ion channel Piezo1. However, in OA cells a significant reduction in Piezo1 expression was observed following treatment with diacerein

Conclusions: Cyclic tensil strain can reduce matrix destroying enzymes, and when used in combination with diacerein, the activity of integrin signalling components is changed. The observed effects can be mainly attributed to diacerein's capacity to modulate STAT3 and Piezo1, which are both potential targets to prevent the progression of $O A$.

Disclosure of Interest: None declared

DOI: 10.1136/annrheumdis-2018-eular.6810

\section{AB0092 IL37 INHIBITS PROTEOGLYCAN LOSS IN HUMAN OA CARTILAGE: LINK BETWEEN IL37 AND MMP3}

E. Van Geffen ${ }^{1}$, A. van Caam ${ }^{1}$, C. Thudium², A.-C. Bay-Jensen ${ }^{2}$, E. Blaney Davidson ${ }^{1}$, P. van der Kraan ${ }^{1} .{ }^{1}$ Rheumatology, Radboud University Medical Center, Nijmegen, Netherlands; ${ }^{2}$ Rheumatology, Nordic Bioscience, Herlev, Denmark

Background: Glycosaminoglycans (GAGs) are essential for the pressure-resistant function of cartilage. During osteoarthritis (OA), GAGs are lost from cartilage. This loss impairs the functional and structural integrity of cartilage, thereby accelerating further cartilage damage. Proteoglycan degradation is mediated by enzymes such as MMP3, MMP13 and ADAMTS5

Objectives: Recently we discovered that interleukin 37 (IL37) lowers the expression of these enzymes in human OA chondrocytes. The goal of this study was to investigate if IL37 protects against GAG loss in freshly obtained human OA explants.

Methods: Human cartilage was obtained from ten OA patients undergoing total knee or hip arthroplasty. Per condition 6 cartilage explants of $4 \mathrm{~mm}$ in diameter were used. Explants were incubated with recombinant IL37 (rhIL37) for up to 6 days. Every other day new rhIL37 was added. Additionally, an MMP3-inhibitor, or MMP13-inhibitor or ADAMTS5-inhibitor was added in the same protocol. Sulfated GAGs (sGAGs) were visualised by histology, and sGAG release in culture medium was quantified using 1,9-dimethylmethylene blue. To study sGAG synthesis, explants were incubated with rhlL37 followed by 4 hour labelling with ${ }^{35} \mathrm{SO}_{4}$. Alternatively, explants were pre-labelled for 4 hour with ${ }^{35} \mathrm{SO}_{4}$ followed by incubation with rhIL37 to study sGAG degradation. Additionally, expression levels of proteoglycans and cartilage matrix degrading enzymes were measured by qPCR and Western Blot. Activity of MMP and ADAMTS enzymes was determined by measuring FFGV, ARGS and NITEGE neo-epitope levels in the supernatant of the cultures, using ELISA.

Results: Culturing human OA explants for up to 6 days, caused a reduction in GAG content as observed by loss of Safranin O staining. In addition, a release of sGAGs was measured in the supernatant of on average $17,5 \mu \mathrm{g} / \mathrm{ml}$ per mg cartilage. Incubation of cartilage explants with rhIL37 significantly reduced the release of GAGs. A maximal reduction of $24 \%$ was already observed with the lowest dose of rhIL37 $(1 \mathrm{ng} / \mathrm{ml})$. No effect of rhIL37 on the amount of incorporated ${ }^{35} \mathrm{SO}_{4}$ was observed, indicating that rhlL37 does not alter the synthesis of GAGs. This is supported by the observation that rhIL37 does not affect the mRNA expression of the large proteoglycans and SLRPS. In contrast, we did find that rhIL37 significantly reduced the loss of ${ }^{35} \mathrm{SO}_{4}$-labelled GAGs from cartilage explants. In addition, rhIL37 reduced MMP3 and MMP13 protein expression and lowered both MMP and ADAMTS mediated degradation of proteoglycan fragments: FFGV neo-epitope and ARGS and NITEGE neo-epitopes, confirming that rhIL37 reduces active degradation of sGAGs. Lastly, to investigate which proteolytic enzyme contributes to the sGAG release in our culture system, a MMP3-, MMP13- or ADAMTS5-inhibitor was added to the explants. Strikingly, we found that only MMP3 inhibition mimicked IL37 function, suggesting that the effects of rhIL37 run via MMP3.

Conclusions: Our data show that rhIL37 reduces sGAG release of cartilage explants, indicating that IL37 supports cartilage matrix integrity. To our knowledge this is the first report demonstrating this anti-destructive effect of IL37 on freshly obtained human OA cartilage explants. Possible these effects run via MMP3 because IL37 reduced MMP3 expression and only MMP3 inhibition results in similar effects as rhIL37 addition.

Disclosure of Interest: None declared

DOI: 10.1136/annrheumdis-2018-eular.6332

\section{AB0093 NATURAL POLYMER-BASED HYDROGELS FOR CARTILAGE REGENERATION}

\section{E. Montell ${ }^{1}$, L. García-Fernández $z^{2}$, A. Torrent ${ }^{1}$, J. San Román ${ }^{2} .{ }^{1}$ Pre-Clinical} RandD Area, Bioibérica, S.A.U., Barcelona; ${ }^{2}$ Biomaterials Group. CIBER's Bioengineering, Biomaterials and Nanomedicine (CIBER-BBN), Institute of Polymer Science and Technology (ICTP-CSIC). Instituto de Salud Carlos III, Madrid, Spain

Background: Cartilage is a tough, flexible tissue found throughout the body. It can become damaged because of a sudden injury, such as a sports injury, or gradual wear and tear (osteoarthritis). Injured articular cartilage has a poor regenerative capability, for this reason, tissue engineering may provide a fundamental therapeutic solution to repair cartilage injury.

Human articular cartilages are composed of dense extracellular matrix and chondrocytes. One of the major components in the extracellular matrix is hyaluronic acid. The combination of hyaluronic acid with other natural polymers to prepare hydrogel scaffolds may be potentially useful in many tissue engineering applications, including cartilage regeneration.

Objectives: We propose the synthesis of hydrogels based on hyaluronic acid and chitosan by a new strategy of synthesis using di-isocyanates to obtain an interpenetrated network of chitosan and hyaluronic acid.

Methods: Different kinds of hydrogels were synthesised using hyaluronic acid (Bioibérica, Spain), natural polymers (gelatine, chitosan) and stabilised with diisocianates. The hydrogels were charged with chondroitin sulfate (Bioibérica, Spain). Morphological Characterisation: The hydrogels were characterised by scanning electron microscopy and energy dispersive X-ray microscopy in order to study the morphology and the composition of the hydrogels.

Hydrogel Swelling: Dynamic swelling experiments were performed by placing discs of stabilised hydrogels in PBS at $37.0^{\circ} \mathrm{C}$, and measuring their weight gain as a function of time.

Biocompatibility: Cytotoxicity in vitro assays (MTT) and cell adhesion tests (Alamar Blue) were performed on the hydrogels using different cell lines to corroborate the biocompatibility of the hydrogels.

Results: All the synthesised hydrogels presented a high interconnected porosity. The studied hydrogels swelled around $200 \%$. After five hours equilibrium was reached and this equilibrium depended on the ratio hyaluronic acid/natural polymer increasing with the amount of hyaluronic acid present on the hydrogel. MTT assay indicated that the extracts collected at different times from the hydrogels were not cytotoxic and could be considered biocompatible. Cell adhesion assay demonstrated that the hydrogels supported cell adhesion and proliferation, and this behaviour was better on SC-loaded hydrogels.

Conclusions: Hydrogels based on hyaluronic acid and natural polymers are proposed as scaffolds for tissue engineering. The presence of interconnected porous and the good swelling properties, as well as the biocompatibility, represent a great advantage for application in cartilage regeneration. The addition of drugs or glyco saminoglycans is being studied to improve the cartilage regeneration.

Disclosure of Interest: None declared

DOI: 10.1136/annrheumdis-2018-eular.3089

\section{AB0094 POSSITIVE EFFETCS OF CHIROPRACTIC MANIPULATION ON SUBCHONDRAL BONE MINERAL DENSITY, CARTILAGE DAMAGE AND SYNOVIAL INFLAMMATION IN OSTEOARTHRITIC RABBITS}

F.M. Conesa ${ }^{1}$, R. Fujikawa ${ }^{2}$, A. Mediero ${ }^{1}$, P. Gratal ${ }^{1}$, F. Mulero $^{3}$, G. HerreroBeaumont $^{1}, \mathrm{R}$. Largo ${ }^{1}, \mathrm{~A}$. Ortega-de Mues ${ }^{1,2}$. ${ }^{1}$ IIS-Fundación Jiménez Díaz-UAM; ${ }^{2}$ Madrid College of Chiropractic-RCUEMC; ${ }^{3}$ Centro Nacional de Investigaciones Oncológicas (CNIO), Madrid, Spain

Background: Osteoarthritis $(O A)$ is a degenerative joint disease characterised by the degradation and inflammation of cartilage and synovium with bone damage. Different approaches that decrease subchondral bone remodelling during OA have demonstrated to improve cartilage damage and synovial inflammation. Chiropractic is a therapeutic approach focused on the diagnosis, treatment and prevention of musculoskeletal disorders. Chiropractic manipulation (CM) is essentially manual, allowing the chiropractor to restore the normal range of motion and function of the joints, muscles, and ligaments. We have previously observed 
that $\mathrm{CM}$ is able to increase subchondral bone mineral density (BMD) in an experimental model of osteoporosis ${ }^{1}$

Objectives: To evaluate if $\mathrm{CM}$ could prevent the subchondral BMD alterations induced by $\mathrm{OA}$, in association to an improvement in synovial membrane inflammation and cartilage damage in an OA model in rabbits.

Methods: Ten male New Zealand rabbits were submited knee surgery to induce OA by transection of anterior cruciate ligament. CM was performed using the chiropractic adjusting instrument ActivatorV 3 times a week during 8 weeks as follows: Force 2 setting was applied onto the tibial tubercle of the rabbit right hind limb (CM-OA group), at an angle of $90^{\circ}$, whereas the corresponding left hind limb received a false manipulation (FM-OA group) consisting of ActivatorV firing in the air and touching the tibial tubercle. Three healthy animals were used as controls. Following sacrifice, tibiae and femora were removed for $\mathrm{MCT}$ and histological evaluation. Synovial inflammation was evaluated by Krenn's score and the protein presence of VEGF, MMP3 and CollagenVI in the synovial membrane was evaluated by western blot

Results: In the OA rabbits, subchondral BMD decreased in relation to control animals (OA 4729 \pm 193 vs Control $5181 \pm 209 \mathrm{mg} / \mathrm{cc}$ ), been partially reversed in the tibiae of OA rabbits with CM (TM-OA $5055 \pm 216$ vs FM-OA 4404 $\pm 170 \mathrm{mg} / \mathrm{cc}$ ). Subchondral trabecular bone structural parameters were analysed by microCT and a significant decrease of bone volume/trabecular volume (BV/TV), trabecular number $(\mathrm{TbN})$ and trabecular thickness ( $\mathrm{TbTh}$ ) was observed in the OA rabbits, while trabecular separation (TbS) increased compared to control animals. TM-OA group showed a significant improvement of these parameters compare to FM-OA group. TM-OA had lower cartilage damage compare to FM-OA (TM-OA 4 $\pm 0,67 \mathrm{AU}$ vs FM-OA $8 \pm 1,25 \mathrm{AU}$ ). TM-OA synovial membranes presented a total

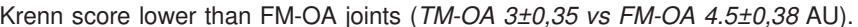
OA synovial membranes showed higher levels of CollagenVI respect to control ones; TM-OA synovial membranes presented less expression of CollagenVI than FM-OA group (TM-OA $1.4 \pm 0,13$ vs FM-OA 2.2 $\pm 0,3 \mathrm{AU}$ ), been this associated with a decrease of both MMP3 (TM-OA 1.2 $\pm 0,1$ vs FM-OA 1.7 $\pm 0.2 \mathrm{AU})$ and VEGF (TM-OA 1.2 \pm 0.14 vs FM-OA 1.9 $\pm 0.26 \mathrm{AU}$ ).

Conclusions: These results support the hypothesis that $\mathrm{CM}$ may ameliorate subchondral BMD alterations induced by $\mathrm{OA}$, in association to an improvement on synoviopathy and cartilage degradation.

\section{REFERENCE:}

[1] López-Herradón A, et al. Impact of Chiropractic Manipulation on Bone and Skeletal Muscle of Ovariectomized Rats.CalcifTissuelnt 2017;101(5):519529.

Disclosure of Interest: F. Conesa: None declared, R. Fujikawa: None declared, A. Mediero Grant/research support from: CP15/00053 PI16/00991, P. Gratal: None declared, F. Mulero: None declared, G. Herrero-Beaumont Grant/research support from: PI16/00065, R. Largo Grant/research support from: PI15/00340, A. Ortega-de Mues: None declared

DOI: 10.1136/annrheumdis-2018-eular.4713

\section{AB0095 ATTEMPT TO DEVELOP A CHIMERIC CO-CULTURE SYSTEM TO DIFFERENTIATE MOUSE OSTEOCLASTS BY CULTURING MOUSE PRECURSOR CELLS WITH HUMAN SYNOVIAL FIBROBLASTS}

H. Yazawa, M. Yanagisawa, Y. Aizaki, Y. Araki, K. Sato, T. Mimura. Department of Rheumatology and Applied Immunology, Saitama Medical University, Saitama, Japan

Background: In rheumatoid arthritis (RA), inflammatory synovial tissue called the pannus proliferates and erodes the articular cartilage and bone in the affected joints. Osteoclasts, multinucleated cells of monocyte/macrophage lineage, are implicated in the bone destruction in RA. Thus, osteoclasts are considered an important therapeutic target in the prevention of the joint destruction. Mouse bone marrow cells differentiate into osteoclasts when co-cultured with osteoblasts or stromal cells in the presence of reagents such as 1,25-dihydroxyvitamin $D_{3}(1,25$ $\left.(\mathrm{OH})_{2} \mathrm{D}_{3}\right)$ and prostaglandin $\mathrm{E}_{2}\left(\mathrm{PGE}_{2}\right)$. There are no osteoblasts in the RA synovium, but there are fibroblasts and various inflammatory cells such as macrophages and lymphocytes. Thus, synovial fibroblasts may function as supporting cells for osteoclastogenesis in place of osteoblasts.

Objectives: The aim of this study was to establish a chimeric co-culture system of osteoclast differentiation using human synovial fibroblasts and mouse monocyte/macrophage lineage cells.

Methods: Synovial tissues were obtained from RA patients who underwent joint replacement surgery. Mouse osteoblasts were obtained from the calvariae of 2- or 3-day-old newborn C57BL/6 (B6) mice. Mouse bone marrow cells were prepared from femoral bones. Osteoclasts were visualised with tartrate-resistant acid phosphatase (TRAP) staining. The protein levels of RANKL and its decoy receptor, osteoprotegerin (OPG), in the culture supernatant were quantified using ELISA.
Results: We confirmed that mouse osteoclasts could be differentiated in vitro by culturing bone marrow cells in the presence of human M-CSF and RANKL. We then cultured mouse osteoclast precursors with human synovial fibroblasts in the presence of $1,25(\mathrm{OH})_{2} \mathrm{D}_{3}$ and $\mathrm{PGE}_{2}$. The murine cells seemed to disappear in the course of the co-culture, whereas they survived when exogenous human M-CSF was added to the system. Interestingly, however, they did not become TRAP-positive multinuclear cells, suggesting that synovial fibroblasts do not provide a sufficient amount of the osteoclast differentiation factor, RANKL. Following these results, we used ELISA to quantify the level of human RANKL and OPG in the culture supernatant of synovial fibroblasts. Predictably, the level of RANKL was below the detection limit with or without the presence of $1,25(\mathrm{OH})_{2} \mathrm{D}_{3}$ and/or $\mathrm{PGE}_{2}$. In contrast, that of OPG was very high, irrespective of the reagents added. We also quantified the levels of mouse RANKL and OPG in the culture supernatant of mouse osteoblasts. As expected, RANKL was detectable in this case Interestingly, the level of OPG was very high and comparable to that of human OPG produced from synovial fibroblasts.
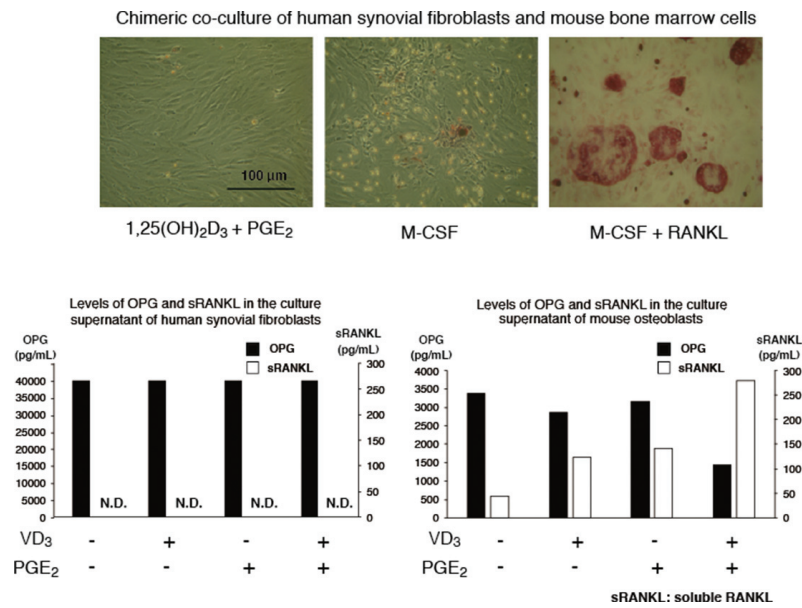

Abstract AB0095 - Figure

Conclusions: Synovial fibroblasts cannot be substituted for osteoblasts in a coculture system of osteoclast differentiation. This is probably because synovial fibroblasts do not provide sufficient RANKL and M-CSF. Instead, they produce a large amount of OPG. This may be a mechanism by which ectopic osteoclastogenesis is inhibited. Thus, the osteoclasts observed in the pannus may be dependent on membrane-bound RANKL from other sources, like lymphocytes, or may be differentiated by stimulation with cytokines other than RANKL, such as TNF- $\alpha$ and IL-6. ${ }^{12}$

\section{REFERENCES}

[1] Yokota K, et al. Arthritis Rheumatol 2014;66:121-9.

[2] O'Brien W, et al. Arthritis Rheumatol 2016;68:2889-2900.

Disclosure of Interest: None declared DOI: 10.1136/annrheumdis-2018-eular.3574

\section{AB0096 EXPRESSION AND FUNCTION OF NEUROPEPTIDE Y RECEPTORS IN HUMAN ARTICULAR CARTILAGE: INFLUENCE OF GENDER AND OSTEOARTHRITIS}

I. Ferreira ${ }^{1}$, C. Sousa ${ }^{2}$, S. Silva ${ }^{1}$, F. Judas ${ }^{3}$, C. Cavadas ${ }^{2}$, A.F. Mendes ${ }^{2} .{ }^{1}$ Center for Neuroscience and Cell Biology, ${ }^{2}$ Faculty Pharmacy and CNC, University of Coimbra; ${ }^{3}$ Orthopaedics Department and Bone Bank, University and Hospital Center of Coimbra, Coimbra, Portugal

Background: Elevated levels of neuropeptide Y (NPY) were reported in osteoarthritic $(O A)$ joints ${ }^{1}$. No information exists regarding the role of NPY in OA joints besides mediating or potentiating nociceptive transmission.

Objectives: To determine whether NPY receptors are present and functional in human chondrocytes, by evaluating the ability of NPY to activate important signalling pathways and to modulate autophagy, which was shown to be induced by this neuropeptide in hypothalamic neurons ${ }^{2}$ and to be a crucial homeostatic mechanism in chondrocytes, whose reduction contributes to OA pathogenesis ${ }^{3}$.

Methods: Immunofluorescence for the NPY receptor subtypes, $Y_{1}, Y_{2}$ and $Y_{5}$, was performed in the human chondrocytic cell line, C28/I2, and in human cartilage sections obtained from multi-organ donors (4 males, 55-75 years old, mean $=67.25$, 3 females, 33-68 years old, mean $=55.33$ ) at the Bone and Tissue 